\title{
ZUR ENGLISCHEN GRAMMATIK.
}

\section{1.}

\section{Die lautgruppen thw- und kw- im Neuenglischen.}

In den Transactions of the Cambridge Philological Society V (1904), s. 197 ff. hat W. W. Skeat Notes on some examples of the occurrence of initial $\mathrm{w}$ in written English veröffentlicht, die darauf ausgehen, einen weitgehenden einflufs des Anglonormannischen auf die englische aussprache nachzuweisen. So hätten die Normannen englisches wulf durch ulf nachgeahmt, das dann seinerseits sich im Englischen festgesetzt hätte. Ebenso wird der übergang von $w h>w$, von $w r$ - $>r$, von th $>d$ (in fiddle, murder, could!!), von thw $>$ th (thwong $>$ thong) erklärt. Skeat hat seine aufstellungen nicht bewiesen. Wo er anglonormannischen einflufs behauptet, handelt es sich um englische lautgesetze.

Hier möchte ich nur auf die lautgruppe thw eingehen, da ihre entwickelung noch nicht klargelegt worden ist. So hat Skeat das verhältnis von thwitel und whittle nicht erläutert. Im anschlufs daran soll auch auf die entwickelung der gruppe $k w$ kurz hingewiesen werden. Mundartliches wik = quick nämlich möchte E. Björkman, Skandinavian LoanWords in Middle English S. 201) vermutungsweise auf skandinavisches vikt, vigt zurückführen; aber da liegt m. e. ebenfalls eine nach englischen lautgesetzen entwickelte form vor.

1. Die lautgruppe thw- hat in englischen mundarten eine ähnliche entwickelung durchgemacht wie $k n$-. Der gruppe $k n$ entsprechen in den heutigen mundarten $k n, t n h-t n, n h, n$ $(n h=$ stimmloses $n)$, vgl. Verf., Gutturallante $\mathbf{s .} 1 \mathrm{ff}$. 
Neben mundarten mit bewahrtem thw finden wir andere, in denen durch den einflufs des anlautenden stimmlosen konsonanten $w$ selbst stimmlos geworden ist. In manchen gegenden ist stimmloses $w h$ weiter verschoben worden $\mathrm{zu}$ stimmhaftem $w: t h w>w h>w$.

Die Shetland- und Orkney-Inseln, die erst in spätneuenglischer zeit das Nordische mit dem Englischen vertauschten (vgl. Noreen in Pauls Grdr. I', 418), haben $b w$ - durch tw- ersetzt. Lautsubstitutionen können wir dort ja häufig beobachten. Vgl. noch chase, cheap mit $\check{s}$ gegenüber tš aller anderen mundarten (Gutturallaute s. 17 f.).

In der schriftsprache finden wir sowohl thw als $w h$. Da steht thwack neben whack 'schlagen'; whittle 'schneiden, messer' neben veraltetem (dialektischem) thwittle (zu ae. puìtan = veraltetem thwite, dial. thwite, white, vgl. E.D.D.). In thwong aus bwang ist $w$ vor dunklem vokal geschwunden: schriftsprachlich thong 'riemen'. Im nordhumbrischen gebiet, im nördlichen mittelland und in gewissen südlichen dialekten (vgl. E. St. XXX, 369 f.) ist altes $a$ vor $x$ nicht verdunkelt worden und deshalb $w$ nicht ausgefallen: dort finden wir heute whan oder was vor. Diese mundartliche form wird von den wörterbüchern als schriftsprachlich verzeichnet: whang (subst.) 'riemenleder', whang (verbum) 'durchpeitschen (vgl. dtsch.-mundartlich verledern), in lange dünne streifen schneiden'. - Für thwart finden wir in der älteren sprache auch whart, vgl. N. E.D. unter overthwart.

2. Ähnlich wie $k n$ - hat sich auch $k w$ - entwickelt: $k w>$ ${ }^{*} k v h>t w(h)>w h>w(w h=$ stimmloses $u) ; \operatorname{vgl}$. E. D. D., N.E.D. unter $Q$ und B. Grüning, Schuund und Zusatz von Konsonanten in den neuenglischen Mundarten, Strafsburger diss. 1904, s. 33. Die verschiedenen stufen der entwickelung sind (wie bei $k n$ ) in den englischen mundarten noch nebeneinander $\mathbf{z u}$ finden.

Die mundart von Windhill hat tw in twil 'quill', twilt 'quilt', twilt (beat, thrash), dagegen $w$ in wik 'quick', in wišin 'cushion' (afrz. cuissin > kuišin = wišin), und kw- in kiwaiat 'quiet', kwaleti 'quality', kwin 'queen' usw.; vgl. Wright $\S 324 \mathrm{f}$. und index. In Windhill haben sich offenbar die lautungen verschiedener mundarten gemischt, und die wörter mit 
$k w$ - werden schriftsprachlich sein. E. Kruisinga, A Grammar of the Dialect of West Somerset, Bonn 1905, s. $76^{5}$ ) greift das eine wort wišin heraus und stellt dafür eine sondererklärung auf: in twišin sei $t$ als artikel aufgefafst und wišin abgetrennt worden. Aber wie erklärt sich das adjektiv oder adwerb wik? Und wie wišin in mundarten, die den artikel in der form $t$ nicht kennen?

\section{II. \\ E. Coote's bemerkungen über englische aussprache} (1596).

In den Stationers' Registers findet sich (nach Arber's Transscripts III, 17) unter dem 18. Dezember 1596 eingetragen: The Englische Scholemaister, teachinge all his schollers of what age soeuer the most easie short and perfect order of distinct readinge and true writinge our Englishe tonge. Verfasser ist Edward (Edmund) Coote, der von 1596--1597 direktor der grammar school in Bury St. Edmund's, Suffolk, war (vgl. Dict. of National Biogr.). Das kleine schulbuch hat viele auflagen erlebt, bis weit in das 18. jahrhundert hinein wurde es neu gedruckt: eine 54. auflage erschien 1737 (vgl. W. C. Hazlitt, Biographical Collections and Notes III, $2^{\text {nd }}$ supplement, s. 20).

Die erste auflage von 1596 ist nur in einem exemplar gefunden worden: das büchelchen (ohne titelblatt) ist im besitz des British Museum. Es wird beschrieben von Hazlitt a. a. o. III, 47.

Der sprachliche teil dieses schulbuches verdient seines hohen alters wegen beachtung. Ich stelle das bemerkenswerte daraus zusammen.

1. S. 22. ... some pronounce these words: blind, find, behind, short, other blinde, behinde with (e) long. - (e) ist offenbar druckfehler für (i). Die aussprache blĭnd, fĭnd, behind stand neben blaind, foind, behaind.

2. S. 24. Unter den wörtern mit stummem $h$ stehen whole (vgl. Luick, Untersuchungen § 85) und authoritie (also noch nicht mit schriftaussprache). 
(gh) Comming together, (except in Ghost) are of most men but little sounded, as might, sight: pronounced as mite, site: but in the end of a word, some countries sound them fully, other not at all, as some say plough, bough, slough, other plow, bou, slou: Thereupon some write burrough, some burrow: but the truest is both to write and pronounce them. Vgl. dazu s. 33, wo gesagt wird, die schreibung might mit gh 'is the truer writing, and it should have a little sound'.

Das ist das früheste grammatikerzeugnis für den schwund der gutturalen spirans (nach schreibungen ist er freilich viel früher anzusetzen), vgl. Gutturallaute s. $78 \mathrm{f}$. Dazu käme jetzt noch der begründer der phonetischen stenographie, John Willis, der in seiner Art of Stenographie, London 1602, die anweisung gibt, die 'dicke aspiration' $g h(h, w, y$ nennt er 'light aspirations') in night u. dgl. wegzulassen.

3. S. 27. (ti) before (on) is pronounced (si) as in redemption, (except $f$, or $x$ goe before, as in question, adustion, mixtion) and commonly before other vowels, as in patience ...

Coote bezeugt also noch nicht $\check{s}$ für $s i$, das zu seiner zeit schon vorhanden war.

4. S. $27 \mathrm{f}$. Von wörtern mit gleicher aussprache, aber verschiedener bedeutung und schreibung werden genannt: reigne - reine - raine, two - to, their - there, wait - weight, nay (not so) - neigh, sunne (sun) - sonne (son), here heare, prophet - profite, I heard - hard, mille wright - write, some - summe. - Von den wörtern mit gleicher aussprache und schreibung, aber verschiedener bedeutung seien genannt: The right eare. Eare thy land, for an eare of corn.

here $=$ hear deutet für das verbum auf me. hẹre ohne einflufs des $r$; vgl. die zeugnisse von Ellis $E$. $E$. $P$. aus dem 16. jahrh.

Das praeteritum hard 'heard' wird uns durch schreibungen und grammatikerzeugnisse wiederholt bezeugt. Primäres (me.) herrde ergab regelrecht harde; heutiges $h^{r} d$ geht auf sekundäres (ne.) hërd(e) zurück; vgl. dialektisches larn neben hochenglischem $\hbar^{r} n$ 'learn'.

Der schwund des gh in weight, wright steht im einklang mit der unter $g h$ gegebenen regel. 
Beachtenswert ist weiter die gleichung their $=$ there. Der diphthong $e i$ war darnach schon $\mathrm{zu} \bar{e}$ geworden. Eine französische grammatik von 1580 (J. B. Gen. Ca., Le maistre d'escole Anglois) bezeugt schon $e$ (wie in frz. estre) für $a i$, ei in affaires, they. Auf zusammenfall von ai und $\bar{a}$ deuten schon die schreibungen whate, wate für wait in den Cely Papers 1479, 1481 (s. 23, 78).

j. Der artikel $t h$ ' vor vokalisch anlautendem substantiv ist bekannt; Coote citiert 'thintent, for the intent, which exactly should be written, th' intent'. Der bestimmte ertikel hatte frühneuenglisch doppelte form: th vor vokal, tha vor kons. th' gehörte der sprache des gewöhnlichen lebens an und war nicht etwa eine poetische freiheit, wie man wohl annimmt (vgl. Schipper, Englische Metrik II, 101 ff., Grundriss der englischen Metrik, § 101). Die vorvokalische form ist von der vorkonsonantischen verdrängt worden, aus dem einfachen grund, weil es mehr substantive mit konsonantischem als mit vokalischem anlaut gibt. Später hat sich das verallgemeinerte the wieder in zwei formen gespalten: thi vor vok., tha vor kons.

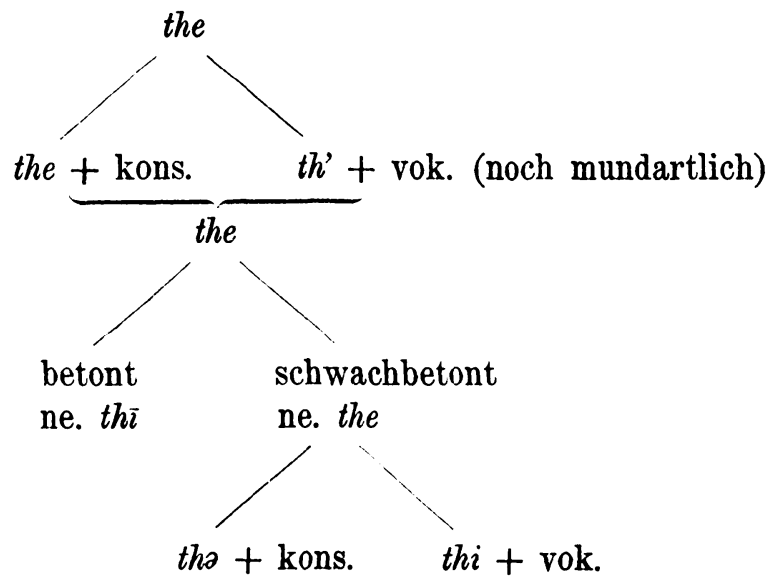

6. Beachtenswert sind besonders Cootes bemerkungen über vulgäre sprache.

Der lehrer sagt zum schüler (s. 30 f.): 
I know not what can easily deceiue you in writing, vnlesse it be by imitating the barbarous speech of your countrie people, whereof I will give you a taste, thereby to offer you an occasion to take heed, not of these onely, but of any like: Some people speake thus: The mell standeth on the hell, for The mill standeth on the hill: so knet vor knit: bredg for bridg: knaw for gnaw: knat for gnat: belk for belch: yerb for herb: griffe for graffe: yelk for yolk: ream for realm: aferd for afraid: durt for dirt, gurt for girth: stomp for stamp: ship for sheep: hafe for halfe: sample for exãmple: parfit for perfect; dauter for daughter: carten for certaine: carchar for carcheife: lease for leash 1): hur for hir: sur and suster for sir and sister: to spat for to spit etc.

So doe they commonly put $v$ for $f$ (die auflagen von 1636 und 1696 verbessern: $f$ for $v$ ) as feale for veale.

Take heed also you put not ... id for ed as vnitid for vnited, which is Scottish. And some ignorantly write a cup a wine for a cup of wine: and other like absurdities.

Coote's liste enthält zunächst einige doppelformen, die auf verschiedenen ursprung hinweisen oder in alte zeit hinaufreichen, wie griffe - graffe (N.E.D.), aferd - affraid, parfit - perfect $\left.{ }^{2}\right)$, yelk - yolk, spit - spat usw.

Im übrigen werden einige dialektische lautwandlungen bezengt. Die auswahl der mundartlichen eigentümlichkeiten ist planlos.

\section{Vokalismus.}

i. Die hinneigung des $i$ zu $e$ war bekanntlich weit verbreitet.

Ist es zufall, dals Coote's wörter mit $e$ für $i$ (mill, hill, knit, bridge) alle ae. $y$ hatten?

$\boldsymbol{i}+\boldsymbol{r}$. Wertvoll ist die mitteilung, dals $i r$ in vulgärer sprache wie ur gesprochen werde. Das ist das erste gram-

1) In der auflage von 1662 umgekehrt.

2) Hier ist einfach die frz. form durch die lat. verdrängt worden. Anders Köppel, Spelling-Pronunciations, s. 9. 
matikerzeugnis für diese verschiebung. Schreibungen weisen auf noch früheren dialektischen wandel von ir $>$ ur: sur $=$ sir, das oft vorkommt, beweist zwar seiner häufig unbetonten stellung wegen nichts, wohl aber z. b. die umgekehrte schreibung Thirsday = Thursday in den Paston Letters 1479 (ed. Gairdner III, 261). ') Der zusammenfall von ir und ur wird dann auch von Hodges in der Primrose für das Hochenglische 1644 bezeugt. Dagegen kennt Mason 1622 (vgl. Brotanek s. XXVI) nur den beginn des lautwandels, die verschiebung von ir $>$ er; ebenso das Alphabet Anglois 1625 unter $i$ :

Quand il est suiuy d'vne $r, \&$ d'vne autre consone apres $r$, se prononce comme un $e$, comme bird, berd: third, therd: first, ferst.

Jones, Phonography 1701, lä[st für ir noch er neben ur zu. Wenn auch ir noch gestattet ist, wie z. b. in virgin (s. 110), handelt es sich um einfluls des schriftbildes auf die aussprache. Man vergleiche dazu die mitteilung von $R$. Nares, Elements of Orthoepy, London 1784, s. 27 *:

It seems that our ancestors distinguished these sounds [ir, ur] more correctly. Bishop Gardiner [1637-1705], in his first letter to Cheke, mentions a witticism of Nicolas Rowley, a fellow Cantab. with him, to this effect: 'Let handsome girls be called virgins, plain ones vurgins'. Si pulchra est, virgo, sin turpis, vurgo vocetur.

Es galt also damals der zusammenfall von ir und ur noch nicht für fein. Auch Walker will einen unterschied gemacht haben (vgl. Einleitung zum Wtbch., § 108):

$i$ in virtue, virgin etc. has exactly the sound of $e \ldots$, which approaches the sound of $u$; but when it comes before $r$, followed by another consonant in a final syllable, it acquires the sound of $u$ exactly.

1) Vgl. auch Georg Neumann, Orthographie der Paston Letters von 1422-1461 (d. h. bd. I und II von Gairdner's ausgabe), Marburger Studien zur engl. Philologie II (1904), s. 31, 66 und 123 und Karl Süssbier, Sprache der ('ely l'apers 1475-1488, Berliner diss. 1905, s. 29 und 92 (manches unsicher!), und Dibelius, Litztg. 1905, sp. 1113. 
Und dem berühmten schauspieler Garrick wurde von einem dr. Hill in einem pamphlet der vorwurf gemacht, er verwechsle $i$ und $u$ : virtue $>$ vurtue (Walker unter virtue).

Auch heute wird ein unterschied zwischen ir und ur von manchen Engländern wenigstens noch angestrebt, vgl. Jespersen, Lehrbuch der Phonetik s. 151 und Storm, Engl. Philologie s. $456^{1}$. Ich weifs nicht, ob diese unterscheidung etwa eine mundartliche unterlage hat.

Im anschlufs daran sei darauf hingewiesen, dals der eigenartige palatale vokal in girl, der früher im Hochenglischen heimisch war (gę̧l, gerl), wohl mit der eben besprochenen aussprache von virgin $=$ verdžin zusammenzustellen ist; vgl. auch Storm s. 453.

Das lautgesetz, wonach sekundäres $\breve{e} r$ und $i r=\bar{\partial} r$ wird, wirkte nur, wenn $r$ im auslaut oder vor kons. stand, also nicht in very, interrogate, irritate, spirit (älteres sperit $=$ afrz. esperit); stirring $=$ stżrin ist selbstverständlich analogiebildung. In ähnlicher weise wird auch die lautgruppe al vor vokal nicht verändert: vgl. fallow (ae. fealu), mallow, sallow; dagegen $a l$ im auslaut $=\bar{\phi} l(a l l$, fall $)$, vor kons. $>\bar{\phi}(l), \bar{a}$ (salt, talk, half).

e. Die aussprache carten 'certain', vor der hier gewarnt wird, hat in frühneuenglischer zeit der gebildeten sprache angehört; Hodges Primrose 1644 sprach noch so.

Unbetontes $-e d>-i d$ wird für schottisch erklärt.

a. stomp für stamp wird auch von Cooper 1685 für dialektisch erklärt (s. 79), vgl. dazu ronk für rank, das Gill 1621 im dialekt der 'Australes' findet (vgl. Jiriczek's Neudruck s. $32^{23}$ ).

hafe $=$ 'half' deutet entweder die vorstufe des heutigen $h \bar{a} f$ oder des mundartlichen hêf, hęif an; vgl. darüber meine demnächst erscheinenden Untersuchungen zur neuenglischen Lautgeschichte, I. abschnitt, 1. kap.

$\bar{e}$. In bezug auf yerb für herb stimmen wieder Coote und Cooper überein (s. 79), und Miege 1688 findet yerb 'pas du bel usage', Jones 1701 'sounded by some'; vgl. dazu Luick, Unter- 
suchungen $\S 242$ und die schreibung yerbe im 15. und 16. jahrh. (N.E.D.: herb).

ship für sheep ist noch mundartlich. Vgl. Shakespeares wortspiele: Two Gentlemen I, 1, 73, Comedy of Errors IV, 1, 93, Love's Labour's Lost II, 1, 89. - John Drummond (teacher of English in Edinburgh), $A$ grammatical Introduction to the Modern Pronunciation and Spelling of the English Tongue, Edinburg 1767 hat sheep und ship in einer liste von gleichlautenden wörtern. - Einen rest der aussprache ship für shī finden wir in der redensart: to lose the ship (ursprünglich sheep, ewe, hog) for a halfpennyworth of tar 'to lose an object, spoil an enterprize or court failure, by trying to save in a small matter of detail' (N.E.D. unter halfpennyworth, b).

\section{Konsonantismus.}

f. Schwund des $f(v)$ in unbetontem of vor konsonantisch anlautendem wort ist bekannt; of tritt schon me. in der form $o$ auf. Die betontere und vorvokalische form ov ist später verallgemeinert worden; die schreibung hat wohl auch dazu beigetragen.

$f$ für $v$ in veal ist schwer zu beurteilen. In gewissen mundarten 1) (vgl. Wright in Pauls Grdr. I', 980 und die interessanten mitteilungen von J. Kjederqvist, The Dialect of Pewsey, London 1903, s. 93 ff.) wurde $f->v$. Handelt es sich in feal für veal um umgekehrte lautgebung, um eine überschriftsprachliche bildung? - Gill bezeugt für den dialekt der 'Australes' $v$ für $f$, 'et contra $f$ pro $v$ ', z. b. in vineger, vicar. Vgl. auch die me. schreibung $f$ in frz. wörtern bei Behrens, Frz. Lehnwörter s. 166.

Auch die auf -er ausgehende nebenform von kerchief (vgl. dazu N.E.D.) war lange allgemein in Hochenglischen gebräuchlich. Wie von Coote wird sie auch von Cooper für dialektisch erklärt (s. 80).

1) Gill 1621 kennt $v$ für $f$ bei den Australes und Orientales (s. 32). Butler 1633 findet diese aussprache 'in the Western partes' (vgl. Index unter $\boldsymbol{F}$ ). 
l. Für lautgesetzliches ream (vgl. Luick, Anglia XVI, 499) wird schon die schriftaussprache realm vorgezogen, die auch Gill 1621 lehrt. Dagegen lälst der stenograph John Willis, The Art of Stenographie, London 1602, in realme $l$ ebenso aus wie in balm.

gh. Dafs die von Coote getadelte sprache nicht durchweg vulgär gewesen ist, zeigt auch die erwähnung von dauter für daughter: das verstummen des $g h$ vor $t$ wird ja von Coote auch für die gute aussprache gestattet. Freilich möchte er dem $g h$ lieber 'a little sound' lassen, aber da teilt er offenbar die scheu der unter dem einflufs des schriftbildes stehenden sprachmeister, einen geschriebenen buchstaben in der aussprache zu unterdrücken. Ähnlich behauptet z. b. Boyer, The Royal Dictionary English and French 1702, in years, pounds sei $s$ fast unhörbar gewesen (»on ne la fait presque point sentir «): in wirklichkeit hiefsen diese pluralformen entweder years, pounds oder year, pound (alte neutrale plurale ohne $-s$ ).

gn. Die ersetzung von gn durch $k n$ ist bemerkenswert. Wie $k n$ - gesprochen wurde, wird nicht gesagt. Unter den stummen buchstaben wird $k$ vor $n$ nicht genannt. Auch Hodges, English Primrose 1644, setzt $g n$ dem $k n$ gleich. Wie aber $k n$ gesprochen wurde, erfahren wir auch von ihm nicht; Hodges schreibt $k n$ ohne zeichen; jedenfalls war $k$ nicht stumm, sonst hätte er $\underline{k} n$ geschrieben. Es ist sehr wohl möglich, daIs gn zunächst zu kn geworden ist, wie im Niederländischen (Pauls Grdr. I', 655).

gnat wird seit dem 14. jahrh. gelegentlich mit $k n$ geschrieben, gnaw seit dem 15. jahrh. (N.E.D.). Das deutet auf frühen zusammenfall von gn und $\mathrm{kn}$.

Das erste mir bekannte phonetikerzeugnis für $g n->n$ ist das des stenographen F. Coles, The newest, plainest, and the shortest Short-hand, London 1674, der unter den 'letters which are not at all, or but little and seldom sounded' (s. 5) $g$ in gnat nennt.

Andere grammatikerzeugnisse für die entwickelung von kn-, gn- sind Gutturallaute s. 4 ff. zusammengestellt. Hinzugefügt sei, dafs Nathanael Strong (school-master in London), Englands Perfect School-Master, London ${ }^{8}$ 1699, darauf auf- 
merksam macht, man solle schreiben gnash not nash, gnat not nat, gnaw not naw, knight not night (s. 79).

Auf früheren übergang von $k n->n$ - deuten gelegentliche schreibungen. Im 16. jahrh. wird gelegentlich $k$ vor $n$ weggelassen, vgl. N.E. D.: knitch, knop, knuckle 'knöchel'; nave statt knave Misogonus II, 5, 82, wozu Brandl, Quellen des weltlichen Dramas vor Shakespeare, QF. LXXX, s. 661 bemerkt: 'weil in zusammensetzung'. Umgekehrt wird $\mathrm{kn}$ - für $n$ - geschrieben: knat für nat 1604 in Nordengland (a. a. 0. s. 7), knouches für nouches 1562 in urkunden aus Lancashire (= afrz. nouche, vgl. N.E.D. unter ouch). Das $N . E . D$. verzeichnet fortknight 16. jahrh. für fortnight, das jedoch auch auf $k n>t n$ weisen könnte.

Unter diesen umständen möchte ich nicht so bestimmt, wie es W. A. Craigie, Athencum 1902, 84 tut, annehmen, dals in Miltons sprache my known und mine own noch nicht gleich lauteten, da kn noch unverändert gewesen sei. Man hatte nämlich im Samson Agonistes I, 218 my known in mine own verwandeln wollen (vgl. dazu Athenoum 1901, 878 und 1902, 50).

\section{III.}

\section{Über einige konjunktionen.}

1. Dafs konjunktionen aus sätzen entstanden sind, ist schon öfters gezeigt worden. J. Wackernagel, Vermischte Beiträge zur griech. Sprachkunde, Univ.-progr. Basel 1897, s. $22 \mathrm{ff}$., hat eine reiche liste solcher konjunktionen zusammengestellt, die durch Ebeling in Vollmöller's Jahresbericht über die Fortschritte der roman. Philologie V. bd., I, 222, 238 noch vermehrt wird. 1) In neuerer zeit haben Körting, Zs. f. frz. Spr. XVIII, 263 ff. und Meyer-Lübke, Romanische Syntax s. 635, bedenken dagegen geltend gemacht. Ihre bemerkungen schliefsen sich an frz. cur an, das man aus dem fragewort quare? abzuleiten gewöhnt ist (vgl. H. Ebel in Kuhns Zs. VI (1856), 206 ff., später L. Tobler, Beitr. V, 377, Wehrmann,

1) Nenerdings leitet Holthausen, $J . F$. XVII, 458 germ. $a k$ aus einem aufforderungssatz (imperativ) ab: $a k=$ lat. age. 
Rom. Stud. V, 436). Körtings einwände habe ich Zs. f. frz. Spr. $\mathrm{XIX}^{2}, 128 \mathrm{zu}$ widerlegen gesucht. Tobler hat Vermischte Beitr. III, 79 von neuem die entstehung von car aus fragendem quare? verfochten. Neuerdings wendet sich Ebeling a. a. o. gegen Körtings und Meyer-Lübkes bedenken. Die schärfste waffe aber hat sich Ebeling nicht zunutze gemacht.

Die beste widerlegung finden solche zweifel an der möglichkeit der entstehung einer begründenden konjunktion aus einem fragewort durch einen hinweis darauf, dafs wir die fragliche erscheinung in lebenden sprachen unmittelbar verfolgen können.

In deutschen mundarten unterbricht der redende nicht selten seine erzählung mit warum, um den grund selbst hinzuzufügen. Er kann nicht kommen, warum - er ist krank. Dieses warum hat häufig keinen frageton. Die pause dahinter ist sehr kurz und fehlt oft ganz.

Sehr schön läfst sich unsere erscheinung auch am Englischen beobachten. Als begründende konjunktion finden wir in der älteren sprache und in heutigen mundarten for why: He cannot come, for why - he is not well. Belege für diesen sprachgebrauch bei Shakespeare s. bei A. Schmidt, Sh.-Lexikon, unter why und Franz, Sh.-Grammatik, § 408. For why in den heutigen mundarten wird oft ohne frageton gesprochen, dahinter ist eine pause mehr oder weniger deutlich wahrzunehmen. In älteren drucken finden wir hinter diesem for why entweder ein fragezeichen oder ein komma oder kein satzzeichen. Da haben wir die ganze entwickelung vor uns. for why ist aus einem selbständigen fragesatz ') zur konjunktion herabgesunken.

Die tatsache steht also fest: es ist rorgekommen, dafs ein fragesatz zur konjunktion geworden ist. Das ist Meyer-Lübke gegenüber zu betonen, der an der möglichkeit dieser entwickelung zweifelt, weil „nicht nur das beziehungsverhältnis von quare verändert wird, sondern, was ihm sehr schwer anzunehmen scheint, der frageton verloren geht". Der einwand ist unberechtigt. Die verschiebung der syntaktischen gliederung ist nichts seltenes. Und der frageton ist auch

1) Franz, Shakespeare-Grammatik a. a. o., spricht von einem 'ellipti s c hen' fragesatz. 
sonst nachweislich verloren gegangen. Vgl. z. b. Tobler III, 193. Ich sehe nicht, wie man eine reihe von nebensätzen erklären will, wenn man die möglichkeit nicht zugibt, dals der frageton verloren geht. So geht beispielsweise Kommt er, so gehe ich mit zurück auf Kommt er? So gehe ich mit. In Wilte dōn mīn wille al, To-morwen $\bar{\imath}$ shal mākien pē fre (Havelok 528) ist der erste satz auch ein ursprünglicher fragesatz. ${ }^{1}$ ) Mätzner, Frz. Grammatik s. 593, erkennt richtig in bedingungssätzen eigentliche fragesätze: Je le placerai selon son mérite, devraisje créer une place pour lui. Der verlust des fragetons beruht auf wirkung der analogie. Der frageton in liommt er? so gehe ich mit ist verloren gegangen, weil daneben andere wendungen standen ohne frageton: Er komme! ich gehe mit; oder Er kommt wohl, ich gehe mit usw. usw. Weiterhin ist aber noch die frage aufzuwerfen, ob der frageton im eingeschobenen fragesatz ursprünglich überhaupt vorhanden gewesen sein mufs. Es gibt auch fragesätze ohne frageton. Nicht in jedem fragesatz wird das ohr des hörers durch hohen ton gereizt. Walther Reichel, Entwurf einer deutschen Betonungslehre (Leipzig 1899), s. 17, bemerkt treffend: „Es gibt auch fragesätze, die heruntergehen ... Dann sind sie erwartet, es liegt nahe, diese frage zu stellen, der hörer versteht die frage deshalb leichter und braucht nicht durch die höhe gespannt zu werden. [Z. B:] 'Wie ist das gekommen.'" Das ist gerade unser fall. Diese betonungsverhältnisse von heute werden auch in der alten sprache gegolten haben.

2. Auch die konjunktion whether 'ob' ist ursprünglich ein fragewort, ein fragesat $z$ gewesen: whether? 'welches von beiden?' I ask you, whether you will come or not hiefs ursprünglich in direkter rede: I ask you: wether? will you come or no (not)? Und I asked him whether it was true hiels anfänglich: I asked him: whether? is it true or no (not)? Die umwandlung der ursprünglichen direkten rede in die heutige indirekte hat C. Stoffel, der in seinen Studies in English (1894), s. 197 ff., die konjunktion whether bespricht, nicht aufgeklärt. Vgl. darüber 0. Behaghel, Die Zeitfolge der

1) Die beschreibung dieses sprachgebrauchs im N.E.D. V, 29 unter if, 7 ist unhistorisch. 
abhängigen Rede im Deutschen, Paderborn 1878 und Der Gebrauch der Zeitformen im konjunktivischen Nebensatz des Deutschen, Paderborn 1899, s. $160 \mathrm{ff}$; man beachte besonders s. 173, wo die 'berichtende form' als zwischenstufe zwischen der direkten und der indirekten rede aufgezeigt wird.

Auf abhängige fragesätze ohne konjunktion hat $\mathrm{Zu}$ pitza, Archiv LXXXVII, 67 f., hingewiesen. She asked herself was it her fault (Ch. Reade, $A$ Woman-Hater II, 81) = she asked herself: was it her fault? ('berichtende form'!). Vgl. auch beispiele bei G. Krüger, Syntax der englischen Sprache (Dresden und Leipzig 1904), § 1695 .

3. Die erkenntnis, dafs über- und unterordnende konjunktionen aus hilfsmitteln der beiordnung hervorgegangen sind, betrachtet man wohl als eine errungenschaft der neuesten zeit. Demgegenüber ist festzustellen, dals manche sprachforscher (oder sprachliebhaber) schon frühe wenigstens eine ahnung von dem entstehen der hypotaxe aus der parataxe gehabt haben. Das darf man wohl sagen von St. Skinner, der 1671 in seinem Etymologicon Linguae Anglicanae die konj. if, 'in agro Linc. gif', von dem verbum gifan ableitet. Diese etymologie fand beifall. Im 18. jahrh. hat besonders John Horne Tooke eine ganze reihe von konjunktionen nach diesem prinzip erklärt. Er hatte aufser Skinner noch andere vorgänger, wie J. Cassander in seinen Criticisms on the Diversions of Purley (1790) nachzuweisen suchte.

Horne Tooke hat seine grammatischen erörterungen zunächst in $A$ Letter to John Dunning, Esq. (London 1778)

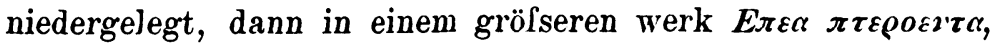
or, the Diversions of Purley (London 1786).

Unser grammatiker spricht es mit grofser entschiedenheit aus, dals die konjunktion that nichts anderes ist als das pronomen that. Durch zweierlei wird diese erkenntnis erschwert (vgl. Letter s. 7 ff.): Unnoticed abbreviation in construction, and difference of position, have caused this appearance of fluctuation. Als beispiel für die erste art (unnoticed abbreviation in construction) führt er u. a. auf: Example: Thieves rise by night, that they may cut men's throats. - Resolution: Thieves may cut men's throats, (for) that (purpose) they rise by night. - Wichtiger und richtiger sind seine erörterungen 
über die zweite art. Ein beispiel dafür (difference of position) ist das folgende: Example: I wish you to believe that I would not wilfully hurt a fly. - Resolution: I would not wilfully hurt a fly, I wish you to believe that (assertion). Und so überall.

Sehr beachtenswert ist, was Horne Tooke von den konjunktionen überhaupt sagt: (s. 16) I deny them to be a separate sort of words, or part of speech by themselves ... the particular signification of each must be sought for from other parts of speech ... So erklärt er if als den imperativ von give, an 'wenn' als den imp. von anan 'to grant', eke als den imp. von eacan 'to add', gin ist das part. von give usw.

Horne's entdeckungen machten grofsen eindruck. Man suchte sie bald in schulbücher einzuführen:

William Hazlitt, $A$ new and improved Grammar of the English Tongue: in which the genius of our speech is especially attended to, and the discoverics of Mi. Horne Tooke and other modern uriters on the formation of our language are, for the first time incorporated. L. 1810. S. VI: The soundest and most useful parts of Mr. T.'s system, are his researches into the origin of indeclinable words, and we have engrafted the result of most of these into our little work. Das ist auf s. $91 \mathrm{ff}$. geschehen.

Einen ähnlichen abschnitt hat W. G. Lewis, A Grammar of the English Language, L. 1821, auf s. $120 \mathrm{ff}$. Auch er hebt auf dem titelblatt hervor, dafs er die discoveries of $M r$. Horne Tooke berücksichtigt.

Noch im jahre 1854 hat Charles Richardson in einem besonderen buch Horne Tooke's lehren verbreitet: On the Study of Language: an Exposition of Eлrce $\pi \tau \varepsilon \cos \nu \tau a$, or the Diversion of Purley by John Horne Toolie (London). Von Hornes buch sagt er: That the work itself ... will live for ever, there can be no doubt.

4. In einem fall kann man auch heute noch die konjunktion nicht ohne die annahme erklären, dals ein verbum darauf eingewirkt habe: ich meine die mundartliche (schottische und nordenglische) form gif für zif, if 'wenn'. Auf diese dialektform hatte schon Skinner bezug genommen. Und Murray, N.E.D. unter gif sagt: Probably it was due to the 
influence of give. Ihm schliefst sich A. Ackermann, Die Sprache der ältesten schottischen Urkunden, Diss. Göttingen 1898, s. 53 an. Auf welche weise der einflufs des verbums sich geltend gemacht haben soll, darüber sprechen sie sich nicht aus. Ich möchte folgende erklärung vorschlagen.

Englisches zive(n), yive(n) ist durch skandinavische einwirkung $\mathrm{zu}$ give(n) geworden. yive und give bestanden eine zeit lang nebeneinander. Nach dem muster von give neben yive hat sich neben altes yif 'wenn' ein neues gif gestellt, das schliefslich in gewissen gegenden alleinherrschend geworden ist. Es würde sich also hier um eine 'analogische lautsubstitution' handeln, die entstehen kann, wenn zwei sprachen oder mundarten sich beeinflussen. Vgl. darüber z. b. Arch. CVII, 414 und Litbl. XXV, 362 (mit weiteren literaturangaben).

Giessen.

Wilhelm Horn. 